\title{
Year in Review 2015: Pediatric ARDS
}

\author{
Ira M Cheifetz MD FAARC
}

\author{
Introduction \\ Pediatric ARDS: Consensus \\ Sedation Management \\ High-Frequency Oscillatory Ventilation \\ Delirium \\ Noninvasive Respiratory Support \\ Lung-Protective Ventilation \\ Adjunct Therapies for the Management of ARDS \\ Summary
}

\begin{abstract}
Led by the work of the Pediatric Acute Lung Injury Consensus Conference, much was published on the topic of pediatric ARDS in 2015. Although the availability of definitive data to the pediatric practitioner for the management of infants and children with pediatric ARDS continues to lag behind that for the adult clinician, 2015 augmented the available medical literature with more information than had been seen for years. This article will review key pediatric ARDS publications with a focus on the Pediatric Acute Lung Injury Consensus Conference consensus definition, sedation management, use of high-frequency oscillatory ventilation, diagnosis of delirium, noninvasive respiratory support, lung-protective ventilation, and adjunct management therapies. Despite the recent progress, additional investigation in each of these areas is essential to the continued advancement of our knowledge and, more importantly, improvements in the outcome for pediatric patients with ARDS. Key words: ARDS, mechanical ventilation, pediatric, surfactant, high-flow oxygen, delirium, sedation, high-frequency ventilation, noninvasive ventilation, corticosteroids. [Respir Care 2016;61(7):980-985. (C) 2016 Daedalus Enterprises]
\end{abstract}

\section{Introduction}

Although representing a relatively small percentage of the total number of pediatric ICU admissions, Children

\footnotetext{
The author is affiliated with the Division of Critical Care Medicine, Department of Pediatrics, Duke Children's Hospital, Duke University Medical Center, Durham, North Carolina.

Dr Cheifetz presented a version of this paper at the Year in Review of the AARC Congress 2015, held November 7-10, 2015, in Tampa, Florida.

Dr Cheifetz has disclosed relationships with Philips and Ikaria.

Correspondence: Ira M Cheifetz MD FAARC, Division of Pediatric Critical Care Medicine, Duke University Medical Center, Box 3046, Durham, NC 27710. E-mail: ira.cheifetz@duke.edu.
}

DOI: $10.4187 /$ respcare. 05017 with ARDS are one of the most challenging patient populations for a clinician to manage. Even more challenging to the pediatric practitioner is the lack of pediatric-specific definitive data to guide clinical management. Until recently, one of the key challenges has been the lack of a pediatric definition for ARDS because pediatric-specific criteria were not included in the initial report by Ashbaugh $^{1}$ in 1967, the 1994 American-European Consensus Conference definition, ${ }^{2}$ or the Berlin definition of 2012. ${ }^{3}$ In 2015, the Pediatric Acute Lung Injury Consensus Conference (PALICC) published pediatric-specific definitions for ARDS and recommendations regarding management and suggested priorities for future research. ${ }^{4}$

This review will focus not only on the definition for pediatric ARDS but on other key related topics as well. The recent clinical trials of high-frequency oscillatory ven- 
Table 1. Definition of Pediatric ARDS by the Pediatric Acute Lung Injury Consensus Conference

\begin{tabular}{|c|c|c|c|c|}
\hline Age & \multicolumn{4}{|c|}{ Exclude patients with perinatal related lung disease } \\
\hline Timing & \multicolumn{4}{|l|}{ Within $7 \mathrm{~d}$ of known clinical insult } \\
\hline Origin of Edema & \multicolumn{4}{|c|}{ Respiratory failure not fully explained by cardiac failure or fluid overload } \\
\hline Chest Imaging & \multicolumn{4}{|c|}{$\begin{array}{l}\text { Chest imaging findings of new infiltrate(s) consistent with acute pulmonary } \\
\text { parenchymal disease }\end{array}$} \\
\hline \multirow{3}{*}{ Oxygenation } & Noninvasive ventilation & \multicolumn{3}{|c|}{ Invasive mechanical ventilation } \\
\hline & PARDS (No severity stratification) & Mild & Moderate & Severe \\
\hline & $\begin{array}{l}\text { Full face-mask bi-level ventilation } \\
\text { or } \mathrm{CPAP} \geq 5 \mathrm{~cm} \mathrm{H} \mathrm{H}_{2}^{2} \\
\qquad \mathrm{PF} \text { ration } \leq 300 \\
\mathrm{SF} \text { ration } \leq 264^{1}\end{array}$ & $\begin{aligned} 4 & \leq \mathrm{OI}<8 \\
5 & \leq \mathrm{OSI}<7.5^{1}\end{aligned}$ & $\begin{array}{c}8 \leq \mathrm{OI}<16 \\
7.5 \leq \mathrm{OSI}<12.3^{1}\end{array}$ & $\begin{aligned} \mathrm{OI} & \geq 16 \\
\mathrm{OSI} & \geq 12.3^{1}\end{aligned}$ \\
\hline \multicolumn{5}{|c|}{ Special Populations } \\
\hline $\begin{array}{l}\text { Cyanotic Heart } \\
\text { Disease }\end{array}$ & \multicolumn{4}{|c|}{$\begin{array}{l}\text { Standard criteria above for age, timing, origin of edema and chest imaging with an } \\
\text { acute deterioration in oxygenation not explained by underlying cardiac disease. }{ }^{3}\end{array}$} \\
\hline $\begin{array}{l}\text { Chronic Lung } \\
\text { Disease }\end{array}$ & \multicolumn{4}{|c|}{$\begin{array}{l}\text { Standard criteria above for age, timing and origin of edema with chest imaging } \\
\text { consistent with new infiltrate and acute deterioration in oxygenation from baseline } \\
\text { which meet oxygenation criteria above. }\end{array}$} \\
\hline $\begin{array}{l}\text { Left Ventricular } \\
\text { Dysfunction }\end{array}$ & \multicolumn{4}{|c|}{$\begin{array}{l}\text { Standard criteria for age, timing and origin of edema with chest imaging changes } \\
\text { consistent with new infiltrate and acute deterioration in oxygenation which meet } \\
\text { criteria above not explained by left ventricular dysfunction. }\end{array}$} \\
\hline
\end{tabular}

Pediatric acute respiratory distress syndrome definition. $\mathrm{OI}=$ oxygenation index, OSI = oxygen saturation index. ${ }^{1} \mathrm{Use}_{\mathrm{P}_{2}}$-based metric when available. If $\mathrm{P}_{\mathrm{aO}}{ }_{2}$ not available, wean $\mathrm{F}_{\mathrm{IO}}$ to maintain $\mathrm{S}_{\mathrm{pO}_{2}} \leq 97 \%$ to calculate OSI or oxygen saturation $/ \mathrm{F}_{\mathrm{IO}_{2}}$ ratio. ${ }^{2}$ For nonintubated patients treated with supplemental oxygen or nasal modes of noninvasive ventilation. ${ }^{3}$ Acute respiratory distress syndrome severity groups stratified by OI or OSI should not be applied to children with chronic lung disease who normally receive invasive mechanical ventilation or children with cyanotic congenital heart disease. $\mathrm{OI}=\left(\mathrm{F}_{\mathrm{IO}_{2}} \times\right.$ mean airway pressure $\left.\times 100\right) / \mathrm{P}_{\mathrm{aO}_{2}}$. OSI $=\left(\mathrm{F}_{\mathrm{IO}_{2}} \times\right.$ mean airway pressure $\left.\times 100\right) / \mathrm{S}_{\mathrm{pO}_{2}}$.

$\mathrm{NIV}=$ noninvasive ventilation

OI = oxygenation index

OSI $=$ oxygen saturation index

$\mathrm{PF}=\mathrm{P}_{\mathrm{aO}} / \mathrm{F}_{\mathrm{IO}_{2}}$

$\mathrm{SF}=\mathrm{S}_{\mathrm{pO}_{2}} / \mathrm{F}_{\mathrm{IO}}$

From Reference 4, with permission

tilation (HFOV) in adult ARDS5,6 have shed a negative light on this ventilatory modality and left pediatric clinicians with a difficult decision on the use of HFOV in a void of pediatric-specific data. A recent publication on HFOV use for pediatric ARDS helps to shed new light on this topic. ${ }^{7}$ Another broad area of increasing interest has become sedation management of the mechanically ventilated pediatric subjects as well as the identification, prevention, and treatment of delirium in this critically ill population. ${ }^{8,9}$ Other related topics that have received increasing attention over the past couple of years are noninvasive ventilation, ${ }^{10}$ high-flow nasal cannula administration, ${ }^{11}$ drive pressure as the potential primary contributor for ventilator-induced lung injury, ${ }^{12}$ corticosteroid therapy for pediatric ARDS, ${ }^{13}$ and exogenous surfactant administration. ${ }^{14}$

\section{Pediatric ARDS: Consensus}

PALICC was a 2-year process that consisted of 27 experts from 8 countries on 3 continents (North America, Europe, and Australia). This interdisciplinary group published their recommendations for the definition of pediatric ARDS in 2015 (Table 1). Some of the key PALICC recommendations included: (1) no age criteria for the definition of pediatric ARDS to better understand the pathophysiology of pediatric ARDS across the spectrum of age groups in future studies, although perinatal-related lung injury was excluded; (2) stratification of the severity of lung injury based on an oxygenation deficit, as defined by the oxygenation index $(\mathrm{OI})$ or oxygen saturation index, if an arterial blood gas was not available; (3) inclusion of infants and children requiring noninvasive ventilation as well as those with congenital heart disease and chronic lung disease; and (4) inclusion of patients with unilateral lung disease.

The severity of pediatric ARDS was based on OI/oxygen saturation index criteria, with pediatric ARDS being described as mild ( $\mathrm{OI}=4-8)$, moderate $(\mathrm{OI}=8-16)$, or severe $(\mathrm{OI}>16) .{ }^{15}$ It should be noted that, similar to the Berlin definition, ${ }^{3}$ PALICC eliminated the previous category of "acute lung injury." The recommendation to allow $\mathrm{S}_{\mathrm{pO}_{2}}$ criteria to define pediatric ARDS was made with the caveat that the $\mathrm{F}_{\mathrm{IO}_{2}}$ be titrated to achieve $\mathrm{S}_{\mathrm{pO}_{2}}$ of $88-97 \%$. PALICC included children receiving noninvasive ventilation (minimum CPAP/expiratory positive airway pressure of $5 \mathrm{~cm} \mathrm{H}_{2} \mathrm{O}$ ) in the pediatric ARDS definition by use of 
the $\mathrm{P}_{\mathrm{aO}} / \mathrm{F}_{\mathrm{IO}_{2}}$ or $\mathrm{S}_{\mathrm{pO}_{2}} / \mathrm{F}_{\mathrm{IO}_{2}}$ ratio to recognize the increasing use of noninvasive ventilation and allow for the possibility of earlier intervention for those with pediatric ARDS. Similar to the prior adult-based definitions, PALICC included the onset of pediatric ARDS within 7 days of a known clinical insult as well as the presence of respiratory failure not fully explained by fluid overload and/or cardiac failure.

PALICC went beyond defining pediatric ARDS and offered 151 recommendations in a series of articles, also published in June 2015, which covered a wide range of topics, including invasive ventilatory support, noninvasive ventilation, pulmonary-specific ancillary approaches, nonpulmonary treatments, respiratory monitoring, extracorporeal support, and others. ${ }^{16-21}$ Also offered by PALICC was a comprehensive discussion of the epidemiology of pediatric ARDS as well as its associated morbidities and longterm outcomes. ${ }^{22,23}$ The reader is directed to the references provided because a comprehensive discussion of the PALICC recommendations is beyond the scope of this review article.

The PALICC guidelines were developed based on peerreviewed, published data as available. When pediatric data were not available, data from adult patients with ARDS and/or neonates with acute lung injury were used. In the event that no data were available, expert opinion was utilized. The common lack of pediatric ARDS data is probably related to the challenges in conducting randomized clinical trials in pediatric subjects with ARDS, including a relatively low overall incidence of pediatric ARDS, heterogeneity in physiology and patient age, subjectivity of the definition of pediatric ARDS (before PALICC), and a lack of consensus for standardized approaches for mechanical ventilation strategies and adjunctive therapies.

If accepted by the pediatric critical care community, the PALICC definition of pediatric ARDS will probably facilitate prompt recognition and diagnosis of pediatric ARDS in clinical practice, improve prognostication and stratification of disease severity to help guide management strategies, and aid in the design of randomized, controlled trials. Multi-center data will be required to validate (or refute) the 2015 PALICC pediatric ARDS definition across all pediatric age groups as well as the management recommendations offered.

\section{Sedation Management}

One of the most challenging aspects of mechanical ventilation for the pediatric patient with acute lung injury is sedation management. On the one hand, pharmacologic sedation is generally required to help ensure the safety and comfort of mechanically ventilated infants and children. On the other hand, the clinician must minimize the adverse effects of such medications, which may include limiting spontaneous ventilation, prolonging the course of mechanical ventilation, inducing critical illness neuromyopathy, increasing the risk for delirium, and causing the development of iatrogenic withdrawal syndromes when sedation is no longer necessary. ${ }^{24-26}$

The RESTORE (Sedation Management for Pediatric Patients with Acute Respiratory Failure) study ${ }^{9}$ was a multicenter cluster-randomized clinical trial that studied the effect of a nurse-implemented, goal-directed sedation management protocol on clinical outcomes in infants, children, and adolescents with acute respiratory failure requiring invasive positive-pressure ventilation. In this largest randomized controlled trial in pediatric acute respiratory failure, 2,449 subjects were enrolled, 50\% from intervention sites and $50 \%$ from control sites. Protocolized sedation did not reduce the length of mechanical ventilation; however, those in the intervention group demonstrated a greater number of awake days and fewer days of opioid administration as well as fewer categories of sedatives prescribed. An increase in adverse events compared with subjects receiving usual sedation management was not seen despite these subjects being more awake.

Although a negative study from the perspective of the primary end point, RESTORE $^{9}$ is important to the pediatric critical care community. The safety of a bedside-driven sedation protocol was demonstrated. More importantly, it showed that mechanically ventilated pediatric subjects can safely be maintained in a more awake state. Such an approach may lead to a reduction in the occurrence of iatrogenic withdrawal because the intervention group had a significantly lower need for methadone administration. Last, it should be mentioned that the RESTORE algorithm did not include dexmedetomidine, which has become more commonly used in the pediatric critical care environment since the initiation of the RESTORE investigation. ${ }^{27,28}$ Further study of a bedside-driven sedation protocol that includes the use of dexmedetomidine should be considered.

\section{High-Frequency Oscillatory Ventilation}

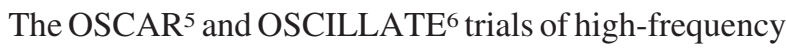
oscillatory ventilation in adult subjects with ARDS have shed a negative light on this ventilatory modality. The OSCAR trial reported no difference in all-cause mortality between HFOV- and conventional mechanical ventilationmanaged subjects. ${ }^{5}$ The OSCILLATE trial was prematurely discontinued due to increased mortality in the HFOV group ${ }^{6}$ However, with a void of definitive pediatric data, clinicians have been left with a difficult decision regarding the use of HFOV for pediatric ARDS. A recent publication on HFOV use for pediatric ARDS helps to shed new light on this topic ${ }^{7}$ by using prospectively collected data from the RESTORE study. ${ }^{9}$ The RESTORE HFOV study compared the length of mechanical ventilation in pediatric sub- 
jects managed with early HFOV (initiated within $24-48 \mathrm{~h}$ of intubation) as compared with those receiving conventional ventilation or late HFOV by means of a propensity score analysis.

Of the 2,449 subjects enrolled in RESTORE, 353 (14\%) were managed at some time during their ventilatory course with HFOV. Two hundred ten (59\%) had HFOV initiated within 24-48 h of intubation. The propensity score model predicting the probability of receiving early HFOV included 1,064 subjects $(n=181$ early HFOV vs $n=883$ conventional mechanical ventilation/late HFOV) with moderate to severe pediatric ARDS based on the PALICC criteria $(\mathrm{OI} \geq 8)$. The most significant contributor to the propensity score model was the degree of hypoxia. After adjusting for risk category, early HFOV was associated with a longer duration of ventilation but not mortality as compared with those managed with conventional mechanical ventilation/late HFOV.

An important question remains: Is the increased length of ventilation with early HFOV use as seen in the RESTORE HFOV study related to HFOV per se or to the manner in which this approach is employed? On the one hand, lowtidal volume ventilation during conventional mechanical ventilation, as has been increasingly employed over recent years, may simply be better than HFOV. However, it must be noted that the general management approaches to mechanical ventilation may differ with HFOV as compared with conventional mechanical ventilation. Specifically, clinicians seem less hesitant to wean conventional ventilator settings than mean airway pressure during HFOV based on a fear that lung de-recruitment will occur in the HFOV setting. Additionally, endotracheal tube suctioning is generally performed less frequently with HFOV with uncertain sequelae.

The HFOV RESTORE study seems to have raised more questions than it has answered. However, the data provided may help in the design of future studies of HFOV for pediatric ARDS. In the meantime, one may speculate that HFOV has a role in the management of pediatric ARDS at the time of peak lung injury followed by aggressive weaning as lung recovery occurs and an earlier transition back to conventional ventilation than has traditionally occurred.

\section{Delirium}

Delirium is the behavioral manifestation of acute cerebral dysfunction associated with an underlying medical illness and presents with acute, fluctuating changes in a patient's mental status. This clinical entity has been wellreported in the adult critical care environment and is associated with increased mortality, morbidity (both short- and long-term), and health-care costs..$^{29-31}$ Recently, increased recognition of this important clinical entity has occurred in the pediatric critical care setting..$^{8,32}$

In a 2015 single-center observational study, ${ }^{8}$ the incidence of delirium in the pediatric ICU setting was demonstrated to be $21 \%$. Risk factors were identified as need for mechanical ventilation, developmental delay, and age between 2 and $5 \mathrm{y}$. Beyond the need for additional research to better characterize pediatric delirium and identify additional predisposing risk factors is the need to learn more in regard to optimal treatment options. In a retrospective review, quetiapine, ${ }^{32}$ an atypical antipsychotic that has been used for the treatment of delirium in the adult critical care setting, was shown to be safe in a cohort of pediatric subjects with ICU delirium. Further investigation, preferably in a prospective fashion, is clearly needed.

\section{Noninvasive Respiratory Support}

Much attention has been paid to noninvasive ventilation in the pediatric population over the past couple of years. Unfortunately, despite the rapid growth in the use of noninvasive ventilation in the pediatric population, definitive data to support its use remain limited. A recent pilot, prospective, randomized clinical trial ${ }^{10}$ comparing noninvasive ventilation and standard oxygen therapy in children $<3$ y of age with respiratory failure after extubation showed no difference between the groups; however, the study was limited by a small sample size and a large number of excluded subjects. This report is important to note because it demonstrates the existence of equipoise (despite the rapidly growing use of noninvasive ventilation) and the ability to study noninvasive ventilation in a randomized, controlled fashion.

Similar to noninvasive ventilation, the use of high-flow nasal cannula continues to increase in the pediatric critical care environment, again without definitive data. Although pediatric studies are limited, it is important to note a recent publication of high-flow oxygen therapy in acute hypoxemic respiratory failure in adult subjects. ${ }^{11}$ Three hundred ten subjects with acute hypoxemic respiratory failure, defined as a $\mathrm{P}_{\mathrm{aO}} / \mathrm{F}_{\mathrm{IO}_{2}}$ ratio of $\leq 300$, without hypercapnia, were randomly assigned to high-flow oxygen via a nasal cannula, standard oxygen delivery by a face mask, or noninvasive ventilation. Intubation rates among the 3 groups did not differ; however, ventilator-free days at day 28 were higher, and mortality at $90 \mathrm{~d}$ was lower in the high-flow nasal cannula group. Although important findings, it should be noted that the primary end point did not show a difference, and this study was limited to those $\geq 18$ y old. A systematic investigation of the use of high-flow nasal cannula support for acute respiratory distress/failure in the pediatric population is needed. 


\section{Lung-Protective Ventilation}

Although lung-protective ventilation has been a focus of ARDS management since the ARDS Network publication on low-tidal volume ventilation in $2002,{ }^{33}$ there has been much discussion regarding the more important mechanism for ventilator-induced lung injury: barotrauma (ie, elevated peak/plateau airway pressures) or volutrauma (ie, increased tidal volume). Although pediatric data are only observational, ${ }^{34-36}$ it is important to review the recent publication by Amato et $\mathrm{al}^{12}$ describing the association between the driving pressure and survival in adults with ARDS. Among ventilation parameters, the drive pressure (difference between peak inspiratory pressure and positive end-expiratory pressure) was most strongly associated with survival. A 1-SD increment in drive pressure (approximately $7 \mathrm{~cm} \mathrm{H}_{2} \mathrm{O}$ ) was associated with increased mortality with a relative risk of $1.4(\mathrm{CI} 1.31-1.51, P<.001)$. Although the pediatric practitioner must be cognizant of the inherent physiologic and anatomic differences between the adult and pediatric lung, the findings of this study cannot be ignored. Until more definitive pediatric data are available, one should consider reducing drive pressure when ventilating the pediatric patient with ARDS.

\section{Adjunct Therapies for the Management of ARDS}

PALICC provides a comprehensive review of the current state of pulmonary and non-pulmonary adjunct therapies for the management of pediatric ARDS. ${ }^{16,17}$ Of particular interest are 2 studies from 2015 that focus on corticosteroid administration and use of exogenous surfactant.

Yehya et al $^{13}$ performed a single-center, observational study in a prospectively enrolled cohort of children with pediatric ARDS. The authors demonstrated that corticosteroid exposure for $>24 \mathrm{~h}$ was independently associated with fewer ventilator-free days at $28 \mathrm{~d}$ and a longer duration of mechanical ventilation even after adjustment for the primary potential confounding variables. It must be noted that as an observational study, it is impossible to distinguish cause and effect from association because confounding variables are likely. This study should be viewed as hypothesis-generating for future prospective, randomized investigations rather than definitive.

In yet another study, the administration of exogenous surfactant was found to have no benefit for adult subjects with ARDS. ${ }^{14}$ The findings of the adult CARDS (Calfactant in Acute Respiratory Distress Syndrome) trial are similar to those of the previous pediatric surfactant study. ${ }^{37} \mathrm{In}$ both studies, exogenous surfactant administration did not improve oxygenation or outcome (hospital stay or survival). At this point, it would seem reasonable to conclude that exogenous surfactant cannot be recommended for rou- tine clinical use for ARDS in either the adult or pediatric populations.

\section{Summary}

In 2015, the medical literature was greatly augmented with regard to the field of pediatric ARDS. The advance of available information was largely led by the work of PALICC as well as the RESTORE investigators. Despite the recent progress, additional investigation in each of the areas discussed in this review article is essential to the continued advancement of our knowledge and, more importantly, improvements in the outcome for pediatric patients with ARDS. It is hopeful that the publications discussed here as well as the topics of research proposed by PALICC will stimulate further basic science, translational, and clinical investigations to continue the advancement of the fields of pediatric respiratory care and pediatric critical care medicine.

\section{REFERENCES}

1. Ashbaugh DG, Bigelow DB, Petty TL, Levine BE. Acute respiratory distress in adults. Lancet 1967;2(7511):319-323.

2. Bernard GR, Artigas A, Brigham KL, Carlet J, Falke K, Hudson L, et al. The American-European Consensus Conference on ARDS: definitions, mechanisms, relevant outcomes, and clinical trial coordination. Am J Respir Crit Care Med 1994;149(3 Pt 1):818-824.

3. ARDS Definition Task Force, Ranieri VM, Rubenfeld GD, Thompson BT, Ferguson ND, Caldwell E, Fan E, Camporota L, Slutsky AS. Acute respiratory distress syndrome: the Berlin Definition. JAMA 2012;307(23):2526-2533.

4. Pediatric Acute Lung Injury Consensus Conference Group. Pediatric acute respiratory distress syndrome: consensus recommendations from the Pediatric Acute Lung Injury Consensus Conference. Pediatr Crit Care Med 2015;16(5):428-439.

5. Young D, Lamb SE, Shah S, MacKenzie I, Tunnicliffe W, Lall R, et al. High-frequency oscillation for acute respiratory distress syndrome. N Engl J Med 2013;368(9):806-813.

6. Ferguson ND, Cook DJ, Guyatt GH, Mehta S, Hand L, Austin P, et al. High-frequency oscillation in early acute respiratory distress syndrome. N Engl J Med 2013;368(9):795-805.

7. Bateman ST, Borasino S, Asaro LA, Cheifetz IM, Diane S, Wypij D, et al. Early high-frequency oscillatory ventilation in pediatric acute respiratory failure: a propensity score analysis. Am J Respir Crit Care Med 2016;193(5):495-503.

8. Silver G, Traube C, Gerber LM, Sun X, Kearney J, Patel A, Greenwald B. Pediatric delirium and associated risk factors: a single-center prospective observational study. Pediatr Crit Care Med 2015;16(4): 303-309.

9. Curley MA, Wypij D, Watson RS, Grant MJ, Asaro LA, Cheifetz IM, et al. Protocolized sedation vs. usual care in pediatric patients mechanically ventilated for acute respiratory failure: a randomized clinical trial. JAMA 2015;313(4):379-389.

10. Fioretto JR, Ribeiro CF, Carpi MF, Bonatto RC, Moraes MA, Fioretto $\mathrm{EB}$, Fagundes DJ. Comparison between noninvasive mechanical ventilation and standard oxygen therapy in children up to 3 years old with respiratory failure after extubation: a pilot prospective randomized clinical study. Pediatr Crit Care Med 2015;16(2):124-130. 
11. Frat JP, Thille AW, Mercat A, Girault C, Ragot S, Perbet S, et al. High-flow oxygen through nasal cannula in acute hypoxemic respiratory failure. N Engl J Med 2015;372(23):2185-2196.

12. Amato MB, Meade MO, Slutsky AS, Brochard L, Costa EL, Schoenfeld DA, et al. Driving pressure and survival in the acute respiratory distress syndrome. N Engl J Med 2015;372(8):747-755.

13. Yehya N, Servaes S, Thomas NJ, Nadkarni VM, Srinivasan V. Corticosteroid exposure in pediatric acute respiratory distress syndrome. Intensive Care Med 2015;41(9):1658-1666.

14. Willson DF, Truwit JD, Conaway MR, Traul CS, Egan EE. The adult calfactant in acute respiratory distress syndrome trial. Chest 2015; 148(2):356-364.

15. Khemani RG, Smith LS, Zimmerman JJ, Erickson S. Pediatric acute lung injury consensus conference group: pediatric acute respiratory distress syndrome: definition, incidence, and epidemiology: proceedings from the Pediatric Acute Lung Injury Consensus Conference. Pediatr Crit Care Med 2015;16(5 Suppl 1):S23-S40.

16. Rimensberger PC, Cheifetz IM, Pediatric Acute Lung Injury Consensus Conference Group. Ventilatory support in children with pediatric acute respiratory distress syndrome: proceedings from the Pediatric Acute Lung Injury Consensus Conference. Pediatr Crit Care Med 2015;16(5 Suppl 1):S51-S60.

17. Tamburro RF, Kneyber MCJ, Pediatric Acute Lung Injury Consensus Conference Group. Pulmonary specific ancillary treatment for pediatric acute respiratory distress syndrome: proceedings from the pediatric acute lung injury consensus conference. Pediatr Crit Care Med 2015;16(5 Suppl 1):S61-S72.

18. Valentine SL, Nadkarni VM, Curley MAQ, Pediatric Acute Lung Injury Consensus Conference Group. Non-pulmonary treatments for pediatric acute respiratory distress syndrome: proceedings from the Pediatric Acute Lung Injury Consensus Conference. Pediatr Crit Care Med 2015;16(5 Suppl 1):S73-S85.

19. Emeriaud G, Newth CJL, Pediatric Acute Lung Injury Consensus Conference Group. Monitoring of children with pediatric acute respiratory distress syndrome: proceedings from the Pediatric Acute Lung Injury Consensus Conference. Pediatr Crit Care Med 2015; 16(5 Suppl 1):S86-S101.

20. Essouri S, Carroll C, Pediatric Acute Lung Injury Consensus Conference Group. Non-invasive support and ventilation for pediatric acute respiratory distress syndrome: proceedings from the Pediatric Acute Lung Injury Consensus Conference. Pediatr Crit Care Med 2015;16(5 Suppl 1):S102-S110.

21. Dalton HJ, Macrae DJ, Pediatric Acute Lung Injury Consensus Conference Group. Extracorporeal support in children with pediatric acute respiratory distress syndrome: proceedings from the Pediatric Acute Lung Injury Consensus Conference. Pediatr Crit Care Med 2015;16(5 Suppl 1):S111-S117.

22. Quasney MW, López-Fernandex YM, Santschi M, Watson RS, Pediatric Acute Lung Injury Consensus Conference Group. The outcomes of children with pediatric acute respiratory distress syndrome: proceedings from the Pediatric Acute Lung Injury Consensus Conference. Pediatr Crit Care Med 2015;16(5 Suppl 1):S118-S131.

23. Flori H, Dahmer MK, Sapru A, Quasney MW, Pediatric Acute Lung Injury Consensus Conference Group. Comorbidities and assessment of severity of pediatric acute respiratory distress syndrome: proceed- ings from the Pediatric Acute Lung Injury Consensus Conference. Pediatr Crit Care Med 2015;16(5 Suppl 1):S41-S50.

24. Randolph AG, Wypij D, Venkataraman ST, Hanson JH, Gedeit RG, Meert KL, et al. Effect of mechanical ventilator weaning protocols on respiratory outcomes in infants and children: a randomized controlled trial. JAMA 2002;288(20):2561-2568.

25. Anand KJ, Willson DF, Berger J, Harrison R, Meert KL, Zimmerman J, et al. Tolerance and withdrawal from prolonged opioid use in critically ill children. Pediatrics 2010;125(5):e1208-e1225.

26. Tobias JD. Tolerance, withdrawal, and physical dependency after long-term sedation and analgesia of children in the pediatric intensive care unit. Crit Care Med 2000;28(6):2122-2132.

27. Hayden JC, Breatnach C, Doherty DR, Healy M, Howlett MM, Gallagher PJ, Cousins G. Efficacy of $\alpha 2$-agonists for sedation in pediatric critical care: a systematic review. Pediatr Crit Care Med 2016;17(2):e66-e75.

28. Whalen LD, Di Gennaro JL, Irby GA, Yanay O, Zimmerman JJ. Long-term dexmedetomidine use and safety profile among critically ill children and neonates. Pediatr Crit Care Med 2014;15(8): 706-714.

29. van den Boogaard M, Schoonhoven L, Evers AW, van der Hoeven JG, van Achterberg T, Pickkers P. Delirium in critically ill patients: impact on long-term health-related quality of life and cognitive functioning. Crit Care Med 2012;40(1):112-118.

30. Pisani MA, Kong SY, Kasl SV, Murphy TE, Araujo KL, Van Ness $\mathrm{PH}$. Days of delirium are associated with 1-year mortality in an older intensive care unit population. Am J Respir Crit Care Med 2009; 180(11):1092-1097.

31. Barr J, Fraser GL, Puntillo K, Ely EW, Gélinas C, Dasta JF, et al. American College of Critical Care Medicine clinical practice guidelines for the management of pain, agitation, and delirium in adult patients in the intensive care unit. Crit Care Med 2013;41(1):263306.

32. Traube C, Silver G, Kearney J, Patel A, Atkinson TM, Yoon MJ, et al. Cornell assessment of pediatric delirium: a valid, rapid, observational tool for screening delirium in the PICU. Crit Care Med 2014; 42(3):656-663.

33. The Acute Respiratory Distress Syndrome Network. Ventilation with lower tidal volumes as compared with traditional tidal volumes for acute lung injury and the acute respiratory distress syndrome. N Engl J Med 2000;342(18):1301-1308.

34. Erickson S, Schibler A, Numa A, Nuthall G, Yung M, Pascoe E, et al. Acute lung injury in pediatric intensive care in Australia and New Zealand: a prospective, multicenter, observational study. Pediatr Crit Care Med 2007;8(4):317-323.

35. Khemani RG, Conti D, Alonzo TA, Bart RD 3rd, Newth CJ. Effect of tidal volume in children with acute hypoxemic respiratory failure. Intensive Care Med 2009;35(8):1428-1437.

36. Zhu YF, Xu F, Lu XL, Wang Y, Chen JL, Chao JX, et al. Mortality and morbidity of acute hypoxemic respiratory failure and acute respiratory distress syndrome in infants and young children. Chin Med J 2012;125(13):2265-2271

37. Willson DF, Thomas NJ, Tamburro R, Truemper E, Truwit J, Conaway $\mathrm{M}$, et al. Pediatric calfactant in acute respiratory distress syndrome trial. Pediatr Crit Care Med 2013;14(7):657-665. 